\title{
Consenso | Protocolo Brasileiro para Infecções Sexualmente Transmissíveis 2020: doença inflamatória pélvica
}

doi: 10.1590/S1679-4974202100011.esp1

\author{
Brazilian Protocol for Sexually Transmitted Infections 2020: pelvic inflammatory disease \\ Protocolo Brasileño para Infecciones de Transmisión Sexual 2020: enfermedad inflamatoria pélvica
}

\begin{abstract}
Maria Luiza Bezerra Menezes' - (D) orcid.org/0000-0001-7001-2005
Paulo Cesar Giraldo ${ }^{2}$ - (1) orcid.org/0000-0003-4365-9879

lara Moreno Linhares ${ }^{3}$ - (1) orcid.org/0000-0002-7846-6885

Neide Aparecida Tosato Boldrini ${ }^{4}$ - (1) orcid.org/0000-0003-1140-5057

Mayra Gonçalves Aragón ${ }^{5}$ - (1) orcid.org/0000-0001-6631-1790

${ }^{1}$ Universidade de Pernambuco, Departamento Materno-Infantil, Recife, PE, Brasil

2Universidade Estadual de Campinas, Departamento de Tocoginecologia, Campinas, SP, Brasil

${ }^{3}$ Universidade de São Paulo, Departamento de Obstetrícia e Ginecologia, São Paulo, SP, Brasil

${ }^{4}$ Universidade Federal do Espírito Santo, Departamento de Ginecologia e Obstetrícia, Vitória, ES, Brasil

${ }^{5}$ Ministério da Saúde, Secretaria de Vigilância em Saúde, Brasília, DF, Brasil
\end{abstract}

\section{Resumo}

0 tema doença inflamatória pélvica está contemplado no Protocolo Clínico e Diretrizes Terapêuticas para Atenção Integral às Pessoas com Infecções Sexualmente Transmissíveis, publicado pelo Ministério da Saúde do Brasil em 2020. A doença inflamatória pélvica é a infecção aguda do trato genital superior feminino decorrente da ascensão canalicular de microrganismos cervicovaginais endógenos e, principalmente, os de transmissão sexual. Entre os agentes etiológicos envolvidos, destacam-se Chlamydia trachomatis e Neisseria gonorrhoeae. As sequelas mais importantes são dor pélvica crônica, infertilidade e gravidez ectópica. 0 diagnóstico clínico apresenta-se como a abordagem prática mais importante. 0 tratamento com antibióticos deve ser iniciado imediatamente diante da suspeição clínica. Descrevem-se orientações para gestores e profissionais de saúde sobre testes diagnósticos, tratamento preconizado, seguimento, aconselhamento, notificação, manejo de parcerias sexuais e de populações especiais. Com a maior disponibilidade da técnica de biologia molecular no Brasil, recomenda-se o rastreio de $C$. trachomatis e $N$. gonorrboeae como estratégia preventiva da doença.

Palavras-chave: Infecção Pélvica; Dor Pélvica; Gravidez Ectópica; Infertilidade; Chlamydia trachomatis; Neisseria gonorrhoeae.

\footnotetext{
Endereço para correspondência:
}

Maria Luiza Bezerra Menezes - Universidade de Pernambuco, Departamento Materno-Infantil, Rua Visconde de Mamanguape $\mathrm{s} / \mathrm{n}$, Encruzilhada, Recife, PE, Brasil. CEP: 52030-010

E-mail: luiza.menezes.24.09@gmail.com 


\section{Apresentação}

0 presente artigo aborda a doença inflamatória pélvica, tema que compõe o Protocolo Clínico e Diretrizes Terapêuticas (PCDT) para Atenção Integral às Pessoas com Infecções Sexualmente Transmissíveis (IST), publicado pela Secretaria de Vigilância em Saúde, do Ministério da Saúde do Brasil. Para a elaboração do PCDT, são realizadas a seleção e a análise das evidências disponíveis na literatura e discussão em um painel de especialistas. 0 referido PCDT foi aprovado pela Comissão Nacional de Incorporação de Tecnologias no Sistema Único de Saúde (Conitec) ${ }^{1}$ e atualizado pelo grupo de especialistas em IST em 2020.

\section{A doença inflamatória pélvica é uma das mais importantes afecções sexualmente transmissíveis, sendo na grande maioria das vezes uma das principais consequências das cervicites.}

\section{Aspectos epidemiológicos}

Denomina-se doença inflamatória pélvica a síndrome clínica inflamatória e infecciosa decorrente da ascensão de microrganismos do trato genital inferior (vagina e colo do útero) para o trato genital superior, podendo comprometer endométrio, tubas, ovários, peritônio pélvico e estruturas adjacentes. Como consequência, podem ocorrer endometrite, salpingite, ooforite e pelviperitonite, dependendo do grau de acometimento. A propagação ocorre predominantemente por via canalicular. ${ }^{2,3}$

Embora a definição clássica de doença inflamatória pélvica inclua apenas a disseminação canalicular e espontânea de microrganismos, não sendo associada a procedimentos cirúrgicos ou gestação, ${ }^{3}$ na atualidade consideram-se também a inserção de dispositivo intrauterino (DIU), biópsia de endométrio, curetagem, entre outros, como responsáveis pela síndrome. ${ }^{2}$

A doença inflamatória pélvica é uma das mais importantes afecções sexualmente transmissíveis, sendo na grande maioria das vezes uma das principais consequências das cervicites. Estima-se um caso de doença inflamatória pélvica para cada oito a dez casos de mulheres com cervicites por Chlamydia trachomatis. ${ }^{3}$ A falta de rápido diagnóstico e tratamento, ou o tratamento inadequado dos casos da doença, aumentam o risco de severas complicações, com consequências extremamente negativas para a saúde da mulher, além dos custos econômicos e sociais. As principais sequelas implicam infertilidade, gravidez ectópica e dor pélvica crônica. ${ }^{4-6}$ Observou-se que, após sete anos do primeiro episódio, 21,3\% das mulheres apresentaram recorrência, 19\% desenvolveram infertilidade e 42,7\% referiram dor pélvica crônica.

Os dados de prevalência existentes são subestimados, já que a doença inflamatória pélvica não é de notificação compulsória e, portanto, o número de mulheres acometidas é desconhecido. Além disso, muitos casos apresentam-se com sintomas clínicos leves ou escassos e passam despercebidos. ${ }^{8}$ Estudos indicam que $10 \%$ a $40 \%$ das mulheres com cervicite por $N$. gonorrhoeae ou C. trachomatis desenvolvem doença inflamatória pélvica. ${ }^{9}{ }^{10}$ No Brasil, a real prevalência do agravo é desconhecida. Por meio do Sistema de Informações Hospitalares do Sistema Único de Saúde, obteve-se o número de internações de mulheres com doença inflamatória pélvica no período de janeiro de 2005 a agosto de 2006, sendo que a média de internações por ano foi de 45.343 casos. Todavia, é importante ressaltar que tais dados refletem apenas os casos graves da síndrome, os quais necessitaram de atendimento hospitalar, e representam apenas pequena parcela das mulheres acometidas, já que a grande maioria apresenta a infecção com sintomas leves e moderados ou são assintomáticas. ${ }^{11}$

Em estudo realizado nos Estados Unidos, 4,4\% de 1.171 mulheres sexualmente ativas entre 18 e 44 anos afirmaram ter tido sintomas sugestivos de doença inflamatória pélvica. ${ }^{12}$ A partir desse resultado, estimou-se que, no período de 2013 a 2014, 2,5 milhões de mulheres tiveram doença inflamatória pélvica nos Estados Unidos. ${ }^{13}$ Outro estudo sugere que ocorrem 800.000 casos anuais da doença nesse país. ${ }^{14}$ Sutton et al. (2005) estimaram 1,2 milhões de consultas por ano por doença inflamatória pélvica nos países desenvolvidos. ${ }^{15}$

Classicamente, o canal endocervical é considerado barreira protetora do trato genital superior. A infecção endocervical com patógenos sexualmente transmissíveis rompe essa barreira e fornece às bactérias vaginais acesso aos órgãos genitais superiores, infectando 0 
endométrio, depois a endossalpinge, o córtex ovariano, o peritônio pélvico e 0 estroma subjacente. ${ }^{2,3,16}$ As razões pelas quais as bactérias do trato genital inferior causam doença inflamatória pélvica em apenas algumas mulheres não são totalmente conhecidas, mas podem estar relacionadas às variações genéticas, à menstruação retrógrada, à resposta imune, à carga bacteriana dos patógenos e às oscilações hormonais do ciclo menstrual, tendo em vista que o muco cervical menstrual tem menor efeito bacteriostático. ${ }^{17-19} \mathrm{~A}$ progressão da infecção por agentes aeróbios determina maior consumo de oxigênio e diminuição do potencial de oxirredução local que, aliados à desvitalização de tecidos, proporcionam ambiente de microaerofilia ou mesmo de anaerobiose (teoria de Monif). ${ }^{16}$ Nesse ambiente, os microrganismos que atingiram o trato genital superior passam a uma fase de crescimento lento e ocorre o desenvolvimento de agentes anaeróbios oportunistas. Como resultado, obtém-se uma condição infecciosa polimicrobiana. ${ }^{2,3,16}$

A partir da observação de que dois terços das mulheres com IST não tinham história anterior ou tratamento de doença inflamatória pélvica, foi proposto o conceito de doença inflamatória pélvica subclínica, que é tão comumente encontrada quanto a doença clínica e apresenta as mesmas etiologias. ${ }^{6,20}$
A maioria dos casos é decorrente de agentes patogênicos sexualmente transmitidos, como $N$. gonorrboeae e $C$. trachomatis. ${ }^{7,21,22}$ Uma minoria de casos agudos não são transmitidos sexualmente, mas associados a germes que colonizam o trato genital inferior ou entéricos, como Mycoplasma hominis e Ureaplasma urealyticum, Peptococcus spp., Peptoestreptococcus spp., Bacteroides spp., Escherichia coli, Streptococcus agalactiae e Campylobacter spp., além de patógenos respiratórios (por exemplo, Haemophilus influenzae, Streptococcus pneumoniae, estreptococos do grupo A e Staphylococcus aureus) ${ }^{23-27}$ Os aeróbios facultativos da microbiota são considerados agentes causadores potenciais. ${ }^{27} \mathrm{Na}$ Figura 1, estão listados agentes etiológicos de doença inflamatória pélvica.

A utilização de métodos de cultura para a identificação de microrganismos tem possibilitado um melhor entendimento da composição da microbiota vaginal saudável, constituída, na maioria das vezes, por uma ou mais espécies de Lactobacillus, que atuam na proteção do meio ambiente vaginal. ${ }^{28}$ Estados de desequilíbrio, como a vaginose bacteriana, que compreende redução ou ausência dos Lactobacillus sp. e aumento nas concentrações e variabilidade de microrganismos anaeróbios, como Gardnerella vaginalis e Mycoplasma sp., podem contribuir para a migração

\begin{tabular}{|c|c|}
\hline Origem dos microrganismos & Agentes etiológicos \\
\hline \multirow{4}{*}{ Microrganismos sexualmente transmissíveis } & Chlamydia trachomatis \\
\hline & Neisseria gonorrhoeae \\
\hline & Mycoplasma genitalium \\
\hline & Trichomonas vaginalis \\
\hline \multirow{10}{*}{ Microrganismos não sexualmente transmissíveis } & Mycoplasma hominis \\
\hline & Ureaplasma urealyticum \\
\hline & Bacteroides spp. e fragilis \\
\hline & Peptoestreptococcus spp. \\
\hline & Prevotella spp. \\
\hline & Escherichia coli \\
\hline & Gardnerella vaginalis \\
\hline & Haemophilus influenzae \\
\hline & Streptococcus spp. e agalactieae \\
\hline & Atopobium vaginae \\
\hline
\end{tabular}

Fonte: adaptado do Protocolo Clínico e Diretrizes Terapêuticas para Atenção Integral às Pessoas com Infecções Sexualmente Transmissíveis, 2020.34

Figura 1 - Agentes etiológicos de doença inflamatória pélvica 
de microrganismos para o trato genital superior. Já foi demonstrado que a vaginose bacteriana aumenta em duas vezes o risco de doença inflamatória pélvica. ${ }^{18,29,30}$

Embora 0 isolamento de microrganismos no trato genital superior tenha sido, até o momento, associado com estados de doença, estudos utilizando métodos moleculares têm demonstrado a presença de microrganismos no endométrio de mulheres saudáveis, incluindo espécies de Lactobacillus, Mycoplasma hominins, G. vaginalis e Enterobacter sp., entre outros. $^{31,32}$ Entretanto, embora mulheres saudáveis possam abrigar tais microrganismos, o papel destes nos estados de saúde e doença ainda não é bem conhecido. Provavelmente, as interações entre 0 agente infeccioso e a imunidade do trato genital sejam determinantes da permanência no estado saudável ou na alteração para o estado de doença. Adicionalmente, técnicas moleculares têm identificado novos microrganismos, como Atopobium vaginae, e estudos também identificaram novas espécies bacterianas e novos gêneros no trato genital superior de mulheres com doença inflamatória pélvica. Assim, precisam ser esclarecidos os questionamentos sobre a existência de uma microbiota saudável no trato genital superior, e sobre a possibilidade de que os mecanismos que predispõem ao desequilíbrio da flora vaginal fisiológica também predisponham à infecção. Certamente, tais respostas irão levar a indagações sobre quais seriam as melhores abordagens para o diagnóstico e tratamento da doença inflamatória pélvica, assim como as maneiras mais eficazes para atuar na sua prevenção. ${ }^{33}$

Os fatores de risco para doença inflamatória pélvica incluem: ${ }^{34}$ 1) faixa etária, pois adolescentes com múltiplos parceiros sexuais, devido a fatores biológicos e comportamentais próprios dessa fase, apresentam risco três vezes maior de desenvolver doença inflamatória pélvica aguda quando comparadas às mulheres acima de 25 anos, ${ }^{35,36}$ independentemente de escolaridade e renda familiar; 2) comportamento sexual com parcerias múltiplas, início precoce das atividades sexuais e novas parcerias; ${ }^{35} 3$ ) utilização de DIU, pois as mulheres usuárias desse dispositivo apresentam risco ligeiramente aumentado de doença inflamatória pélvica nos primeiros 20 dias da inserção, independentemente do tipo de DIU inserido - de cobre ou com liberação de levonorgestrel. ${ }^{37}$ Esse risco é reduzido nas mulheres tratadas para IST antes da inserção do DIU..$^{38}$

\section{Aspectos clínicos}

0 diagnóstico clínico continua sendo a abordagem mais importante na doença inflamatória pélvica, apesar do amplo espectro de apresentações clínicas. Cerca de $65 \%$ dos casos podem ser oligossintomáticos e assintomáticos e apresentarem, mais tarde, infertilidade por fator tubário. ${ }^{39} 0$ curso temporal é tipicamente agudo, desenvolvendo-se por vários dias; no entanto, pode ocorrer apresentação mais extensa por semanas ou meses. Os sintomas, quando presentes, podem incluir febre, dor abdominal, dor pélvica, dispareunia, corrimento vaginal e disúria ou polaciúria. ${ }^{39,40}$ 0bserva-se sangramento uterino anormal (sangramento pós-coital, sangramento intermenstrual e menorragia) em um terço ou mais dos casos. 0 aparecimento recente de dor abdominal ou pélvica, ou ambas, que se intensifica durante o coito ou durante movimentação vigorosa, pode ser o único sintoma. 0 início da dor durante ou logo após a menstruação é particularmente sugestivo. ${ }^{41}$ Apenas uma minoria desenvolve peritonite ou abscesso pélvico, que geralmente se manifestam por dor mais intensa, maior sensibilidade a exame e características sistêmicas, como febre. ${ }^{41-43}$ Mais raramente, ocorre extensão até a cápsula hepática, causando peri-hepatite (síndrome de Fitz-Hugh Curtis) ou septicemia, ou ambas. ${ }^{27,44}$

0 exame clínico deve incluir aferição de sinais vitais; exame abdominal; exame especular vaginal, com inspeção do colo de útero para friabilidade (sangramento fácil) e corrimento mucopurulento cervical; toque vaginal bimanual, com mobilização do colo; e palpação dos anexos (ovários e trompas uterinas). ${ }^{42}$

As sequelas são dor pélvica crônica, gravidez ectópica e infertilidade. Cerca de $25 \%$ das mulheres com doença inflamatória pélvica terão dor pélvica crônica, $10 \%$ a $50 \%$ terão infertilidade e $15 \%$ a $60 \%$ terão gravidez ectópica, geralmente causada por cicatrizes e aderências nas trompas de Falópio ${ }^{7,45,46}$ Essas proporções geralmente aumentam com o número de episódios de infecções, sendo muito altas em partes da África, Ásia e América do Sul, onde os cuidados de saúde não são facilmente acessíveis. ${ }^{47,48}$ Há também relatos de que a doença inflamatória pélvica pode estar associada a um maior risco de acidente vascular cerebral, câncer de ovário e obstrução aguda do intestino delgado. ${ }^{47-50}$ 


\section{Diagnóstico}

0 diagnóstico clínico de suspeição de doença inflamatória pélvica é realizado a partir da presença de três critérios maiores associados a um critério menor ou um critério elaborado, ${ }^{34}$ apresentados na Figura 2.

Os exames laboratoriais e de imagem auxiliam no diagnóstico e na avaliação de gravidade e são importantes em casos oligossintomáticos. No entanto, em caso de alta suspeição clínica, o tratamento não deve ser retardado.

Os seguintes exames laboratoriais são recomendados: hemograma completo; velocidade de hemossedimentação; proteína $\mathrm{C}$ reativa; exame bacterioscópico para vaginose bacteriana; cultura de material de endocérvice com antibiograma; biologia molecular para $N$. gonorrhoeae e $C$. trachomatis no material de endocérvice, uretra, laparoscopia ou punção do fundo de saco posterior; exame qualitativo de urina e urocultura, para descartar infecção do trato urinário; hemocultura; teste de gravidez, para descartar gravidez ectópica; e exames de imagem. ${ }^{51}$ Exames sorológicos de $C$. trachomatis não são recomendados para o diagnóstico de doença inflamatória pélvica.

A ultrassonografia pélvica é o exame de imagem preferencial, por ser acessível e não invasivo, principalmente para avaliar possíveis complicações relacionadas, como abscesso tubo-ovariano, e para afastar diagnósticos diferenciais. ${ }^{52} \mathrm{Na}$ doença inflamatória pélvica, o principal achado ecográfico de suspeição é uma fina camada líquida preenchendo a trompa, com ou sem líquido livre na pelve. Exames de tomografia e ressonância podem auxiliar em diagnósticos diferenciais de peritonite. ${ }^{42,51}$

A laparoscopia apresenta-se como recurso acurado no diagnóstico de salpingite e possibilita diagnóstico bacteriológico mais completo. No entanto, não detecta endometrite e inflamação tubária menos intensa, e assim não se justifica o seu uso rotineiro na fase inicial da doença, pela baixa sensibilidade ${ }^{18,19}$ e pela morbidade associada.${ }^{41-43} \mathrm{~A}$ laparoscopia teve seu grande mérito durante a década de 1980, por ter suscitado a classificação diagnóstica de Gainesville, ${ }^{53}$ na prática, ainda é bastante utilizada de acordo com os achados clínicos e ultrassonográficos, sendo importante para nortear 0 tratamento, a saber: grau I - salpingite sem peritonite; grau II - salpingite com peritonite; grau III - presença de complexo tubo-ovariano, subdividido em A (hidrossalpinge) e B (abscesso tubo-ovariano); grau IV - abscesso tubo-ovariano roto; e grau $\mathrm{V}$ - qualquer dos graus acima, associado a tuberculose genital.

\begin{tabular}{|c|c|}
\hline Critérios & Descrição \\
\hline \multirow{3}{*}{ Critérios maiores } & Dor no hipogástrico \\
\hline & Dor à palpação dos anexos \\
\hline & Dor à mobilização do colo uterino \\
\hline \multirow{7}{*}{ Critérios menores } & Temperatura axilar $>37,5^{\circ} \mathrm{C}$ ou temperatura retal $>38,3^{\circ} \mathrm{C}$ \\
\hline & Conteúdo vaginal ou secreção endocervical anormal \\
\hline & Massa pélvica \\
\hline & Mais de 10 leucócitos por campo de imersão em material de endocérvice \\
\hline & Leucocitose em sangue periférico \\
\hline & Proteína C reativa ou velocidade de hemossedimentação elevada \\
\hline & Comprovação laboratorial de infecção cervical por gonococo, clamídia ou micoplasmas \\
\hline \multirow{3}{*}{ Critérios elaborados } & Evidência histopatológica de endometrite \\
\hline & Presença de abscesso tubo-ovariano ou de fundo de saco de Douglas em estudo de imagem \\
\hline & Laparoscopia com evidência de doença inflamatória pélvica \\
\hline
\end{tabular}

Fonte: adaptado do Protocolo Clínico e Diretrizes Terapêuticas para Atenção Integral às Pessoas com Infecções Sexualmente Transmissiveis, 2020. ${ }^{34}$

Figura 2 - Critérios diagnósticos de doença inflamatória pélvica 


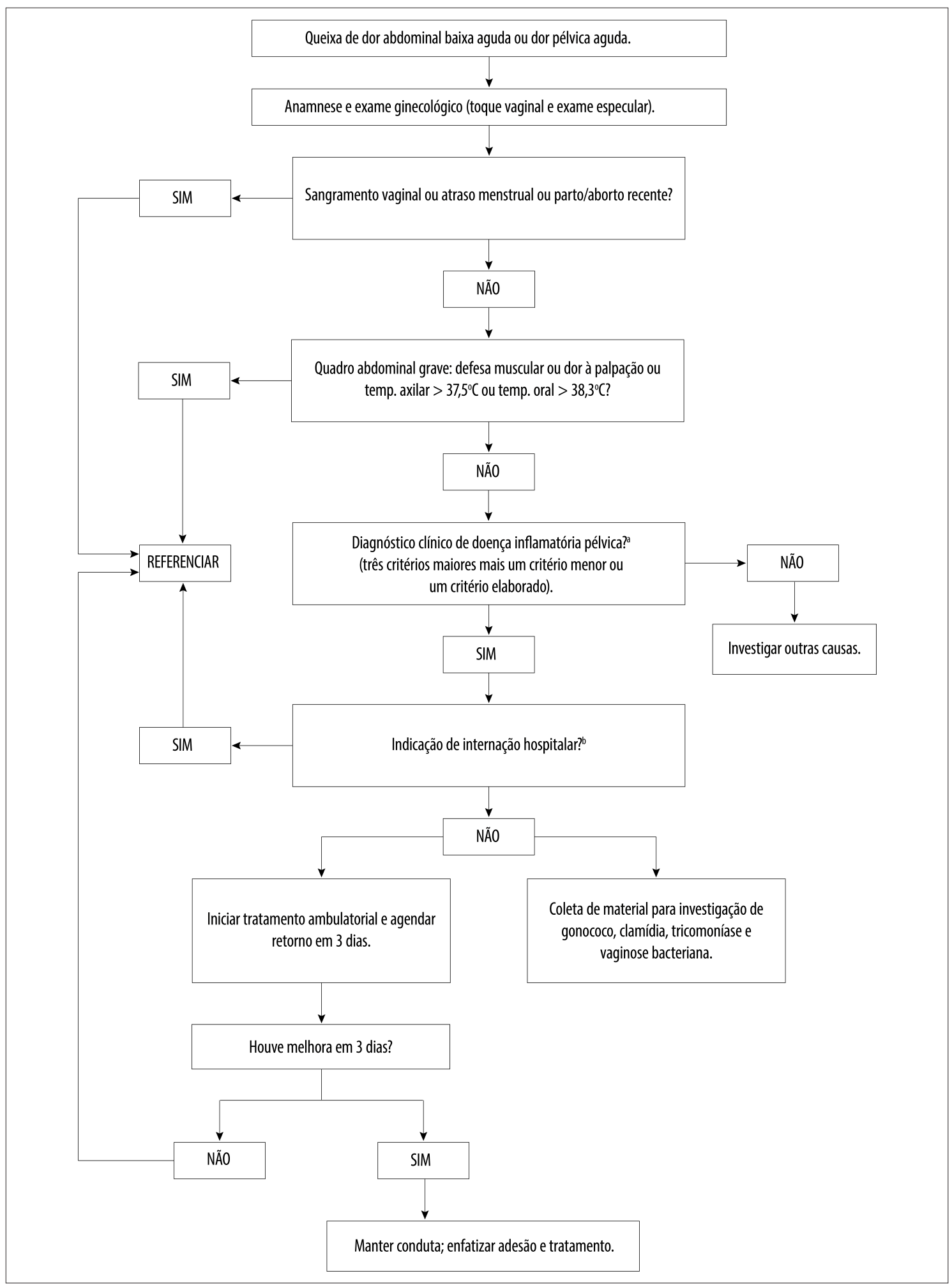

Fonte: adaptado do Protocolo Clínico e Diretrizes Terapêuticas para Atenção Integral às Pessoas com Infecções Sexualmente Transmissiveis, 2020.34

Notas: a) Ver Figura 2 - Critérios diagnósticos de doença inflamatória pélvica; b) Ver Figura 4 - Critérios para indicação de tratamento hospitalar de doença inflamatória pélvica.

Figura 3 - Fluxograma para o manejo clínico de doença inflamatória pélvica 
No diagnóstico diferencial, listam-se gravidez ectópica, apendicite aguda, diverticulite, infecção do trato urinário, litíase ureteral, torção de cisto ovariano ou de mioma uterino, rotura de cisto ovariano, endometriose e endometrioma roto, entre outros. ${ }^{2,16,22}$

\section{Tratamento}

A Figura 3 apresenta as orientações para manejo clínico ambulatorial da doença inflamatória pélvica e indica as necessidades de encaminhamento para avaliação de urgência ou tratamento hospitalar. ${ }^{34}$

0 tratamento deve ser iniciado imediatamente, visando evitar complicações tardias como infertilidade, gravidez ectópica e dor pélvica crônica..$^{54} 0$ tratamento de outras causas comuns de dor pélvica (gravidez ectópica, apendicite aguda, cisto no ovário e dor funcional) dificilmente será prejudicado pela terapia antimicrobiana para doença inflamatória pélvica. ${ }^{42}$ Além de antibióticos, podem ser utilizados analgésicos e anti-inflamatórios para diminuir a sintomatologia.

0 tratamento ambulatorial se aplica às mulheres que apresentam quadro clínico leve sem sinais de pelviperitonite (Estádio I de Gainesville). ${ }^{53}$ 0s demais estádios clínicos e os critérios resumidos na Figura 4 requerem tratamento hospitalar. ${ }^{34}$

0s esquemas terapêuticos devem apresentar cobertura antimicrobiana para os agentes etiológicos de doença inflamatória pélvica, ${ }^{34}$ conforme a Figura 5. A antibioticoterapia deve ser de amplo espectro, ter eficácia contra $N$. gonorrboeae, C. trachomatis e os anaeróbios, em especial 0 Bacteroides fragilis, ainda que não tenham sido confirmados nos exames laboratoriais, e contemplar a vaginose bacteriana, bactérias Gram negativas, bactérias facultativas e estreptococos..$^{55,56}$

Entre os antibióticos de amplo espectro com atividade direcionada para tais agentes, os betalactâmicos, aminoglicosídeos, tetraciclinas, lincosaminas e macrolídeos têm sido bastante estudados. Estes devem ser usados em associação e podem ser administrados por via parenteral ou oral. ${ }^{42,43} 0$ uso parenteral poderá ser suspenso 24 horas após cessarem os sintomas e a terapêutica antimicrobiana por via oral ou intramuscular deve estender-se até 14 dias.

A doxiciclina é 0 agente de escolha para tratar C. trachomatis na maioria dos estudos. A combinação de clindamicina e gentamicina tem atividade moderada contra $N$. gonorrhoeae e $C$. trachomatis in vitro, e o uso de cefalosporinas de segunda geração (cefoxitina ou cefotetana) associadas à doxiciclina apresentam excelentes resultados in vitro. A associação ampicilina/sulbactam é também uma boa escolha. ${ }^{55,56}$ Devido à alta resistência bacteriana, as quinolonas não têm sido usadas para tratar N. gonorrboeae no Brasil. ${ }^{57}$

No caso da doença inflamatória pélvica, o PCDT indica a dosagem de $500 \mathrm{mg}$ de ceftriaxona nos esquemas terapêuticos recomendados. ${ }^{34}$ Essa dosagem foi eleita em decisão da Conitec, com base em avaliação de custo-benefício e de impacto financeiro do uso de ceftriaxona $250 \mathrm{mg}$ em território nacional. ${ }^{58}$ Protocolos internacionais preconizam dose de ceftriaxona de $250 \mathrm{mg}$, conforme disponibilidade do medicamento e evidências locais de susceptibilidade antimicrobiana da N. gonorrboeae. ${ }^{42}$

A melhora clínica deverá acontecer em até três dias após o início do tratamento antimicrobiano. A cura é baseada no desaparecimento dos sinais e sintomas e normalização dos exames laboratoriais de marcadores

\begin{tabular}{|l|}
\hline Critérios para indicação de tratamento hospitalar de doença inflamatória pélvica \\
\hline Abscesso tubo-ovariano \\
\hline Gravidez \\
\hline Ausência de resposta clínica após $72 h$ do início do tratamento com antibioticoterapia oral \\
\hline Intolerância a antibióticos orais ou dificuldades para tratamento ambulatorial \\
\hline Estado geral grave, com náuseas, vômitos e febre \\
\hline Dificuldade na exclusão de emergência cirúrgica (ex.: apendicite, gravidez ectópica) \\
\hline
\end{tabular}

Fonte: adaptado do Protocolo Clínico e Diretrizes Terapêuticas para Atenção Integral às Pessoas com Infecções Sexualmente Transmissíveis, 2020.34

Figura 4 - Critérios para indicação de tratamento hospitalar de doença inflamatória pélvica 


\begin{tabular}{|c|c|c|c|}
\hline Tratamento & Primeira opção & Segunda opção & Terceira opção \\
\hline Ambulatorial & $\begin{array}{l}\text { Ceftriaxona } 500 \mathrm{mg} \text {, intramuscular (IM), } \\
\text { dose única mais doxiciclina } 100 \mathrm{mg}, 1 \\
\text { comprimido, via oral (V0), } 2 x / \text { dia, por } \\
14 \text { dias, mais metronidazol } 250 \mathrm{mg}, 2 \\
\text { comprimidos, V0, 2x/dia, por } 14 \text { dias }\end{array}$ & $\begin{array}{l}\text { Cefotaxima } 500 \mathrm{mg} \text {, IM, dose única mais } \\
\text { Doxiciclina }^{\mathrm{a}} 100 \mathrm{mg}, 1 \text { comprimido, V0, } \\
2 \mathrm{x} / \text { dia, } \text { por } 14 \text { dias, mais metronidazol } \\
250 \mathrm{mg}, 2 \text { comprimidos, V0, } 2 x / \text { dia, por } \\
14 \text { dias }\end{array}$ & \\
\hline Hospitalar & $\begin{array}{l}\text { Ceftriaxona } 1 \mathrm{~g} \text {, intravenoso (IV), } 1 \mathrm{x} / \text { dia, } \\
\text { por } 14 \text { dias mais doxiciclina } 100 \mathrm{mg}, 1 \\
\text { comprimido, V0, } 2 \mathrm{x} / \text { dia, por } 14 \text { dias, mais } \\
\text { metronidazol }^{\mid} 400 \mathrm{mg} \text {, IV, de } 12 / 12 \mathrm{~h}\end{array}$ & $\begin{array}{l}\text { Clindamicina } 900 \mathrm{mg}, \mathrm{IV}, 3 \mathrm{x} / \mathrm{dia} \text {, por } 14 \text { dias } \\
\text { mais gentamicinad (IV ou IM): } 3-5 \mathrm{mg} / \mathrm{kg} \text {, } \\
1 \mathrm{x} / \mathrm{dia} \text {, por } 14 \text { dias }\end{array}$ & $\begin{array}{l}\text { Ampicilina/sulbactam 3g, IV, 6/6h, } \\
\text { por } 14 \text { dias mais doxiciclina } 100 \mathrm{mg}, 1 \\
\text { comprimido, V0, 2x/dia, por } 14 \text { dias }\end{array}$ \\
\hline
\end{tabular}

Fonte: adaptado do Protocolo Clínico e Diretrizes Terapêuticas para Atenção Integral às Pessoas com Infecções Sexualmente Transmissíveis, 2020.34

Notas: a) A doxiciclina é contraindicada durante a gravidez; b) De acordo com avaliação profissional, o metronidazol pode ser descontinuado ou não prescrito em casos leves a moderados, devido à intolerância ao uso e à menor importância de antibioticoterapia para anaeróbios em casos não graves. Orientar quanto ao não uso de bebidas alcoólicas durante e após $24 \mathrm{~h}$ do uso de metronidazol para evitar efeito dissulfiran (antabuse) símile; c) 0 uso parenteral poderá ser suspenso 24 horas após cessarem os sintomas e a terapêutica antimicrobiana por via oral deve se estender até 14 dias; d) A gentamicina pode ser fracionada em 2 a $3 x /$ dia ou dose reduzida quando clinicamente indicado.

\section{Figura 5 - Tratamento da doença inflamatória pélvica}

inflamatórios. Estudo demonstrou que, se a avaliação for feita com critérios bacteriológicos após trinta dias, em $40 \%$ das mulheres ainda persiste a presença de um ou mais agentes bacterianos. ${ }^{7}$ Se houver persistência ou piora do quadro, deve-se considerar rever o diagnóstico. Laparoscopia, ressonância ou tomografia poderão ser realizadas nesses casos. ${ }^{42,51} \mathrm{~A}$ laparotomia está indicada nos casos de massas anexiais não responsivas ao tratamento, ou que se rompem. Poderá ser indicada a culdotomia, caso o abscesso ocupe o fundo de saco de Douglas. Em casos particulares, abscessos tubo-ovarianos podem ser esvaziados com punção guiada por ultrassonografia. ${ }^{34}$

Uma consulta de retorno ao ambulatório para seguimento deve ser programada na primeira semana, sugerindo-se uma segunda consulta 30 dias após a alta hospitalar. No caso de planejamento reprodutivo, deve-se avaliar o uso ou retirada de anticoncepcionais hormonais e do DIU. ${ }^{34,59}$ Recomenda-se abstinência sexual por 30 dias e, após esse prazo, o uso de preservativos em todas as relações sexuais. A remoção do DIU não deve ser, necessariamente, realizada nos casos leves e moderados da doença inflamatória pélvica, ${ }^{41-43}$ mas deve ser considerada caso a usuária deseje fazê-lo ou se não houver melhora clínica após 72 horas da antibioticoterapia, ou, ainda, em casos de doença inflamatória pélvica severa. ${ }^{38}$ Porém, quando indicada, a remoção só deve ocorrer após duas doses do esquema terapêutico. ${ }^{60}$ Nesses casos, deve-se orientar 0 uso de métodos de barreira ou hormonais.

Tanto o episódio agudo da doença inflamatória pélvica quanto suas sequelas têm custos extremamente elevados para as mulheres e para o sistema de saúde. Esses custos têm aumentado substancialmente, tendo em vista que muitas mulheres necessitarão de técnicas de reprodução assistida. ${ }^{61}$

\section{Vigilância, prevenção e controle}

Assim como em outras IST, a prevenção e controle da doença inflamatória pélvica deve incluir 0 aconselhamento centrado na pessoa e suas práticas sexuais, visando seu autorreconhecimento e a minimização de fatores de risco para IST e novos episódios de doença inflamatória pélvica. ${ }^{62}$ Recomenda-se testagem para HIV, sífilis e hepatites B e C. Em casos indicados, deve-se oferecer vacinação contra hepatite $A$, hepatite B e HPV..$^{34}$

Todas as parcerias sexuais devem ser aconselhadas e avaliadas, com oferta de testagem e vacinação. As parcerias sexuais de até dois meses antes do diagnóstico devem ser tratadas empiricamente para $N$. gonorrhoeae e $C$. trachomatis com ceftriaxona $500 \mathrm{mg}$ intramuscular e azitromicina $1 \mathrm{~g}$ via oral em dose única. ${ }^{34}$

A doença inflamatória pélvica não se enquadra na lista de agravos de notificação compulsória do Ministério da Saúde, assim como não se notificam as infecções causadas por $N$. gonorrboeae, C. trachomatis e Mycoplasma genitalium. ${ }^{63}$ Apesar disso, as notificações podem ser realizadas pelo Distrito Federal, estados e municípios, a depender de decisões locais.

A doença inflamatória pélvica é um grande problema de saúde pública. Programas de rastreamento 
para $N$. gonorrhoeae e C. trachomatis demonstraram redução de casos de doença inflamatória pélvica em mulheres. 0 Centro de Controle e Prevenção de Doenças dos Estados Unidos recomenda rastreamento anual, principalmente para mulheres com menos de 25 anos sexualmente ativas e mulheres acima de 25 anos com risco de infecção por $C$. trachomatis, incluindo as respectivas parcerias sexuais. 0 órgão considera, ainda, a possibilidade do rastreio em homens, em cenários com alta prevalência e possibilidades de recursos. ${ }^{42,64}$ No cenário nacional, recomenda-se o rastreamento para $N$. gonorrboeae e $C$. trachomatis em algumas situações: primeira consulta do pré-natal em gestantes com 30 anos ou menos, pessoas com diagnóstico de IST, pessoas vivendo com HIV, situação de violência sexual, pessoas em uso de profilaxia pré-exposição (PrEP) e pós-exposição (PEP) e pessoas com prática sexual anal receptiva sem uso de preservativos. ${ }^{34}$

Uma possibilidade a ser avaliada em protocolos futuros é a inclusão do diagnóstico do $M$. genitalium em mulheres com doença inflamatória pélvica, além da $N$. gonorrhoeae e $C$. trachomatis. ${ }^{65}$ Diretrizes clínicas europeias e norte-americanas incluíram essa investigação em mulheres com doença inflamatória pélvica e homens com uretrites não gonocócicas. Vários estudos apresentaram associação de $M$. genitalium com cervicite e doença inflamatória pélvica; no entanto, não existe evidência de benefícios para rastreamento universal. ${ }^{41-43}$

\section{Referências}

1. Brasil. Ministério da Saúde. Portaria MS/SCTIE n ${ }^{\circ} 42$, de 5 de outubro de 2018. Torna pública a decisão de aprovar o Protocolo Clínico e Diretrizes Terapêuticas para Atenção Integral às Pessoas com Infecções Sexualmente Transmissíveis (IST), no âmbito do Sistema Único de Saúde - SUS [Internet]. Diário Oficial da União, Brasília (DF), 2018 out 8 [citado 2020 out 2]; Seção 1:88. Disponível em: http://bvsms.saude.gov.br/ bvs/saudelegis/sctie/2018/prt0042_08_10_2018.html

2. Ford GW, Decker CF. Pelvic inflammatory disease. Dis Mon [Internet]. 2016 Aug [cited 2020 0ct 2]; 62(8):301-5. Available from: https:// doi.org/10.1016/j.disamonth.2016.03.015

3. Paavonen J. Pelvic Inflammatory disease. From diagnosis to prevention. Dermatol Clin [Internet]. 1998

\section{Populações e situaçõos especiais}

Gestantes com doença inflamatória pélvica têm alto risco de abortamento, corioamnionite e parto prematuro ${ }^{42}$ devendo ser internadas e iniciar imediatamente antibióticos intravenosos de amplo espectro. Doxiciclina e quinolonas são contraindicadas na gestação. ${ }^{34}$

Crianças, adolescentes pré-púberes e mulheres que vivem com HIV, embora possuam maior risco de doença inflamatória pélvica e complicações, têm comportamento clínico geralmente similar e devem ser conduzidas da mesma forma que a população geral. .9,66,67 $^{-1}$

\section{Contribuição dos autores}

Menezes MLB, Giraldo PC, Linhares IM, Boldrini NAT e Aragon MG contribuíram igualmente com a concepção, delineamento, redação e revisão crítica do manuscrito. Todos os autores aprovaram a versão final do trabalho e são responsáveis por todos os seus aspectos, incluindo a garantia de sua precisão e integridade.

\section{Agradecimento}

Os autores agradecem a contribuição para a realização deste trabalho aos membros do grupo técnico de especialistas responsáveis pela elaboração do PCDT para Atenção Integral às Pessoas com IST em 2020.

Oct [cited 2020 0ct 2]; 16(4):747-56. Available from: https://doi.org/10.1016/s0733-8635(05)70041-3

4. Haggerty CL, Gottlieb SM, Taylor BD, Low N, XU F, Ness RB. Risk of sequelae after Chlamydia trachomatis genital infection in women. J Infect Dis [Internet]. 2010 Jun [cited 2020 0ct 2]; 201(Suppl 2):S134-55. Available from: https://doi.org/10.1086/652395

5. World Bank. World development report 1993: investing in health, world development indicators [Internet]. New York: Oxford University Press; 1993 [cited 2020 Jul 20]. Available from: https://openknowledge.worldbank.org/handle/10986/5976

6. Crossman SH. The challenge of pelvic inflammatory disease. Am Fam Physician [Internet]. 2006 Mar [cited 2020 0ct 2]; 73(5):859-64. [update: Am Fam 
Physician. 2006 Dec 15;74(12):2024]. Available from: https://www.aafp.org/afp/2006/0301/p859.html

7. Trent M, Bass D, Ness RB, Haggerty C. Recurrent PID, subsequent STI, and reproductive health outcomes: findings from the PID evaluation and clinical health (PEACH Study). Sex Transm Dis [Internet]. 2011 Sep [cited 2020 0ct 2]; 38(9):879-81. Available from: https:/dx.doi.org/10.1097\%2F0LQ.0b013e31821f918c

8. French CE, Hughes G, Nicholson A, Yung M, Ross JD, Williams T, et al. Estimation of pelvic inflammatory disease diagnoses: trends iNEngland, 2000-2008. Sex Transm Dis [Internet]. 2011 Mar [cited 2020 0ct 2]; 38(3):158-62. Available from: https://doi.org/10.1097/olq.0b013e3181f22f3e

9. Bakken IJ, Ghaderi S. Incidence of pelvic inflammatory disease in a large cohort of women tested for Chlamydia trachomatis: a historical follow-up study. BMC Infect Dis [Internet]. 2009 Aug [cited 2020 0ct 2]; 9:130. Available from: https://doi.org/10.1186/1471-2334-9-130

10. Land JA, Van Bergen JEAM, Morré SA, Postma MJ. Epidemiology of Chlamydia trachomatis infection in women and the cost-effectiveness of screening. Hum Reprod Update [Internet]. 2010 Mar-Apr [cited 2020 0ct 2]; 16(2):189-204. Available from: https://doi.org/10.1093/humupd/dmp035

11. Pinto VM, Szwarcwald CL, Baroni C, Stringati LL, Inocencio LA, Miranda AE. Chlamydia trachomatis prevalence and risk behaviors in parturient women aged 15 to 24 in Brazil. Sex Transm Dis [Internet]. 2011 0ct [cited 2020 0ct 2]; 38(10):957-61. Available from: https://doi.org/10.1097/olq.0b013e31822037fc

12. National Center for Health Statics. National health and nutrition examination survey 2013-2014 [Internet]. Washington, D.C.: Centers for Disease Control and Prevention; 2020 [cited 2020 Jul 22]. Available from: https://www.cdc.gov/nchs/nhanes/index.htm

13. Kreisel K, Torrone E, Bernstein K, Hong J, Gorwitz R. Prevalence of pelvic inflammatory disease in sexually experienced women of reproductive age -United States, 2013-2014. MMWR Morb Mortal Wkly Rep [Internet]. 2017 Jan [cited 2020 0ct 2]; 66(3):80-3. Available from: http://dx.doi.org/10.15585/mmwr.mm6603a3

14. Das Breanne B, Ronda J, Trent M. Pelvic inflammatory disease: improving awareness, prevention, and treatment. Infect Drug Resist [Internet]. 2016Aug [cited 2020 0ct 2]; 9:191-7. Available from: https://dx.doi.org/10.2147\%2FIDR.S91260
15. Sutton MY, Stemberg M, Zaidi A, St Louis ME, Markowitz LE. Trends in pelvic inflammatory disease hospital discharges and ambulatory visits, Unites States, 1885-2001. Sex Transm Dis [Internet]. 2005 Dec [cited 2020 0ct 2]; 32(12):778-84. Available from: https://doi.org/10.1097/01.olq.0000175375.60973.cb

16. Carvalho NS, Takimura M, Von Lisigen R, Freitas B. Doença inflamatória pélvica. In: Fernandes CE, Sá MF. Tratado de ginecologia FEBRASGO. Rio de Janeiro: Elsevier; 2019. p. 287-96.

17. Korn AP, Hessol NA, Padian NS, Bolan GA, Donegan $\mathrm{E}$, Landers DV, et al. Risk factors for plasma cell endometritis among women with cervical Neisseria gonorrhoeae, cervical Chlamydia trachomatis, or bacterial vaginosis. Am J Obstet Gynecol [Internet]. 1998 May [cited 2020 0ct 2]; 178(5):987-90. Available from: https://doi.org/10.1016/s0002-9378(98)70536-8

18. Ness RB, Kip KE, Hillier SL, Soper DE, Stamm $\mathrm{CA}$, Sweet RL, et al. A cluster analysis of bacterial vaginosis associated microflora and pelvic inflammatory disease. Am J Epidemiol [Internet]. 2005 Sep [cited 2020 Oct 2]; 162(6):585-90. Available from: https://doi.org/10.1093/aje/kwi243

19. Morré SA, Karimi O, Ouburg S. Chlamydia trachomatis: identification of susceptibility markers for ocular and sexually transmitted infection by immunogenetics. FEMS Immunol Med Microbiol [Internet]. 2009 Mar [cited 2020 0ct 2]; 55(2):140-53. Available from: https://doi.org/10.1111/j.1574-695x.2009.00536.x

20. Wiesenfeld HC. Pelvic inflammatory disease: treatment in adults and adolescents [Internet]. [S.l.]: UpToDate; 2019 [cited 2020 Jul 22]. Available from: https:/www.uptodate.com/contents/pelvic-inflammatory-disease-treatment-in-adults-and-adolescents

21. Short VL, Totten PA, Ness RB, Astete SG, Kelsey SF, Haggerty CL. Clinical presentation of Mycoplasma genitalium infection versus Neisseria gonorrhoeae infection among women with pelvic inflammatory disease. Clin Infect Dis [Internet]. 2009 Jan [cited 2020 0ct 2]; 48(1):41-7. Available from: https://doi.org/10.1086/594123

22. Sweet RL. Pelvic inflammatory disease: current concepts of diagnosis and management. Curr Infect Dis Rep [Internet]. $2012 \mathrm{Feb}$ [cited 2020 0ct 2]. Available from: https://doi.org/10.1007/s11908-012-0243-y

23. Workowski KA, Berman S; Centers for Disease Control and Prevention (CDC). Sexually transmitted diseases treatment guidelines, 2010. MMWR Recomm Rep 
[Internet]. 2010 Dec [cited 2020 0ct 2]; 59(RR12):1-110. Available from: https://www.cdc.gov/ mmwr/preview/mmwrhtml/rr5912a1.htm?s

24. Haggerty CL, Taylor BD. Mycoplasma genitalium: an emerging cause of pelvic inflammatory disease. Infect Dis Obstet Gynecol [Internet]. 2011 Dec [cited 2020 0ct 2]; 2011:959816. Available from: https://dx.doi.org/10.1155\%2F2011\%2F959816

25. Weinstein SA, Stiles BG. A review of the epidemiology, diagnosis and evidence-based management of Mycoplasma genitalium. Sex Health [Internet]. 2011 Jun [cited 2020 0ct 2]; 8(2):143-58. Available from: https://doi.org/10.1071/sh10065

26. McGowin CL, Anderson-Smits C. Mycoplasma genitalium: an emerging cause of sexually transmitted disease in women. PLoS Pathog [Internet]. 2011 May [cited 2020 0ct 2]; 7(5):e1001324. Available from: https://dx.doi.org/10.1371\%2Fjournal.ppat.1001324

27. Brunham RC, Gottlieb SL, Paavonen J. Pelvic inflammatory disease. NEngl J Med [Internet]. 2015 May [cited 2020 0ct 2]; 372(21):2039-48. Available from: https://doi.org/10.1056/NEJMra1411426

28. Zhou X, Bent SJ, Schneider MG, Davis CC, Islan MR, Forney LJ. Characterization of vaginal microbial communities in adult healthy women using cultivation independent methods. Microbiology (Reading) [Internet]. 2004 Aug [cited 2020 0ct 2]; 150(Pt 8):2565-73. Available from: https://doi.org/10.1099/mic.0.26905-0

29. Galask RP, Larsen B, Ohm MJ. Vaginal flora and its role in disease entities. Clin Obstet Gynecol [Internet]. 1976 Mar [cited 2020 0ct 2]; 19(1):61-81. Available from: https://doi.org/10.1097/00003081-197603000-00008

30. Srinivasan S, Hoffman NG, Morgan MT, Matsen FA, Fiedler TL, Hall RW, et al. Bacterial communities in women with bacterial vaginosis: high resolution phylogenetic analyses reveal relationships of microbiota to clinical criteria. PLoS One [Internet]. 2012 Jun [cited 2020 0ct 2]; 7(6):e37818. Available from: https://doi.org/10.1371/journal.pone.0037818

31. Moller BR, Kritiansen FV, Thorsen P, Frost L, Mogensen SC. Sterility of the uterine cavity. Acta Obstet Gynecol Scand [Internet]. 1995 Mar [cited 2020 0ct 2]; 74(3):216-9. Available from: https://doi.org/10.3109/00016349509008942

32. Kyono K, Hashimoto T, Nagai Y, Sakuraba Y. Analysis of endometrial microbiota by $16 \mathrm{~S}$ ribosomal RNA gene sequencing among infertile patients: asingle-center pilot study. Reprod Med Biol [Internet]. 2018
Jul [cited 2020 0ct 2]; 17(3):297-306. Available from: https://dx.doi.org/10.1002\%2Frmb2.12105

33. Sharma H, Tal R, Clark NA, Segars JH. Microbiota and pelvic inflammatory disease. Semin Reprod Med [Internet]. 2014 Aug [cited 2020 0ct 2]; 32(1):43-9. Available from: https://dx.doi.org/10.1055\%2Fs-0033-1361822

34. Ministério da Saúde (BR). Secretaria de Vigilância em Saúde. Departamento de Doenças de Condições Crônicas e Infecções Sexualmente Transmissíveis. Protocolo clínico e diretrizes terapêuticas para atenção integral às pessoas com infecções sexualmente transmissíveis (IST) [Internet]. Brasília: Ministério da Saúde; 2015 [citado 2020 out 2]. Disponível em: http://www.aids.gov.br/pt-br/ pub/2015/protocolo-clinico-e-diretrizes-terapeuticas-para-atencao-integral-pessoas-com-infeccoes

35. Simms I, Stephenson JM, Mallinson H, Peeling RW, Thomas K, Gokhale R, et al. Risk factors associated with pelvic inflammatory disease. Sex Transm Infect [Internet]. 2006 Dec [cited 2020 Oct 2]; 82(6):452-7. Available from: https:// dx.doi.org/10.1136\%2Fsti.2005.019539

36. Hoenderboom BM, van Benthem BHB, van Bergen JEAM, Dukers-Muijrers NHTM, Götz HM, Hoebe CJPA, et al. Relation between Chlamydia trachomatis infection and pelvic inflammatory disease, ectopic pregnancy and tubal factor infertility in a Dutch cohort of women previously tested for chlamydia in a chlamydia screening trial. Sex Transm Infect [Internet]. 2019 Jun [cited 2020 oct 2]; 95(4):300-6. Available from: https://doi.org/10.1136/sextrans-2018-053778

37. Meirik 0. Intrauterine devices - upper and lower genital tract infections. Contraception [Internet]. 2007 Jun [cited 2020 0ct 2]; 75(Suppl 6):S41-7. Available from: https://doi.org/10.1016/j.contraception.2006.12.017

38. Caddy S, Yudin MH, Hakim J, Money DM; Infectious Disease Committee; Special Contributor. Best practices to minimize risk of infection with intrauterine device insertion. J Obstet Gynaecol Can [Internet]. 2014 Mar [cited 2020 0ct 2]; 36(3):266-74. Available from: https://doi.org/10.1016/s1701-2163(15)30636-8

39. Wiesenfeld HC, Sweet RL, Ness RB, Krohn MA, Amortegui AJ, Hillier SL. Comparison of acute and subclinical pelvic inflammatory disease. Sex Transm Dis [Internet]. 2005 Jul [cited 2020 Oct 2]; 32(7):400-5. Available from: https://doi. org/10.1097/01.olq.0000154508.26532.6a 
40. Jacobson L, Weström L. Objectivized diagnosis of acute pelvic inflammatory disease. Diagnostic and prognostic value of routine laparoscopy. Am J Obstet Gynecol [Internet]. 1969 Dec [cited 2020 Oct 2]; 105(7):1088-98. Available from: https:// doi.org/10.1016/0002-9378(69)90132-X

41. Ross J, Cole M, Evans C, Deirdre L, Dean G, Cousins D. United Kingdom national guideline for the management of pelvic inflammatory disease (2019 interim update) [Internet]. United Kingdom: British association for sexual health and HIV BASHH; 2019 [cited 2020 Jul 22]. Available from: https://www. bashhguidelines.org/media/1217/pid-update-2019.pdf

42. Workowski KA, Bolan GA; Centers for Disease Control and Prevention. Sexually transmitted diseases treatment guidelines, 2015. MMWR Recomm Rep [Internet]. 2015 Jun [cited 2020 0ct 2]; 64(RR03):1-137. Available from: https://www.cdc.gov/ mmwr/preview/mmwrhtml/rr6403a1.htm

43. Ross J, Guaschino S, Cusini M, Jensen J. 2017 European guideline for the management of pelvic inflammatory disease. Int J STD AIDS [Internet]. 2018 Feb [cited 2020 0ct 2]; 29(2):108-14. Available from: https://doi.org/10.1177/09564624177444099

44. Curry A, Williams T, Penny ML. Pelvic inflammatory disease: diagnosis, management, and prevention. Am Fam Physician [Internet]. 2019 Sep [cited 2020 0ct 2]; 100(6):357-64. Available from: https://www.aafp.org/afp/2019/0915/p357.html

45. Weström L, Joesoef R, Reynolds G, Hagdu A, Thompson SE. Pelvic inflammatory disease and fertility. A cohort study of 1,844 women with laparoscopically verified disease and 657 control women with normal laparoscopic results. Sex Transm Dis [Internet]. 1992 Jul-Aug [cited 2020 Oct 2]; 19(4):185-92. Available from: https:// journals.lww.com/stdjournal/Citation/1992/07000/ Pelvic_Inflammatory_Disease_and_Fertility_A.1.aspx

46. Lanjouw E, Ouburg S, Vries HJ, Stary A, Radcliffe K, Unemo M. Background review for the '2015 European guideline on the management of Chlamydia trachomatis infections'. Int J STD AIDS [Internet]. 2015 Nov [cited 2020 0ct 2]. Online ahead of print. Available from: https://doi.org/10.1177/0956462415618838

47. Golden MR, Workowski KA, Bolan G. Developing a public health response to Mycoplasma genitalium. J Infect Dis [Internet]. 2017 Jul [cited 2020 0ct 2]; 216(Suppl 2):420-6. Available from: https://doi.org/10.1093/infdis/jix200

48. Fouks Y, Cohen Y, Tulandi T, Meiri A, Levin I, Almog B, et al. Complicated clinical course and poor reproductive outcomes of women with tubo-ovarian abscess after fertility treatments. J Minim Invasive Gynecol [Internet]. 2019 Jan [cited 2020 0ct 2]; 26(1):162-8. Available from: https://doi.org/10.1016/j.jmig.2018.06.004

49. Fehring RJ, Bouchard T, Meyers M. Influence of contraception use on the reproductive health of adolescents and young adults. Linacre Q [Internet]. 2018 May [cited 2020 0ct 2]; 85(2):167-77. Available from: https://doi.org/10.1177/0024363918770462

50. Haumann A, Ongaro S, Detry 0, Meunier P, Meurisse $\mathrm{M}$. Acute pelvic inflammatory disease as a rare cause of acute small bowel obstruction. Acta Chir Belg [Internet]. 2019 0ct [cited 2020 Oct 2]; 119(5):328-30. Available from: https:// doi.org/10.1080/00015458.2018.1453438

51. World Health Organization - WHO. Diagnóstico laboratorial de doenças sexualmente transmissíveis, incluindo 0 vírus da imunodeficiência humana [Internet]. Brasília: Organização Mundial da Saúde; Ministério da Saúde; 2013 [citado 2020 jul 22]. 255 p. Disponível em: https://www.who.int/reproductivehealth/ publications/rtis/9789241505840/en/

52. Cicchiello LA, Hamper UM, Scoutt LM. Ultrasound evaluation of gynecologic causes of pelvic pain. Obstet Gynecol Clin North Am [Internet]. 2011 Mar [cited 2020 0ct 2]; 38(1):85-114, viii. Available from: https://doi.org/10.1016/j.ogc.2011.02.005

53. Papavarnavas CP, Venter PF, van Staden MJ. Acute salpingitis laparoscopic and microbiological evaluation. S Afr Med J. 1990 Apr;77(8):403-4.

54. Hillis SD, Joesoef R, Marchbanks PA, Wasserheit JN, Cates W Jr, Westrom L. Delayed care of pelvic inflammatory disease as a risk factor for impaired fertility. Am J Obstet Gynecol [Internet]. 1993 May [cited 2020 0cr 2]; 168(5):1503-9. Available from: https://doi.org/10.1016/s0002-9378(11)90790-x

55. Jaiyeoba 0, Lazenby G, Soper DE. Recommendations and rationale for the treatment of pelvic inflammatory disease. Expert Rev Anti Infect Ther [Internet]. 2011 Jan [cited 2020 0ct 2]; 9(1):61-70. Available from: https://doi.org/10.1586/eri.10.156 
56. Duarte R, Fuhrich D, Ross JD. A review of antibiotic therapy for pelvic inflammatory disease. Int J Antimicrob Agents [Internet]. 2015 Sep [cited 2020 0ct 2]; 46(3):272-7. Available from: https:// doi.org/10.1016/j.ijantimicag.2015.05.004

57. Bazzo ML, Golfetto L, Gaspar PC, Pires AF, Ramos MC, Franchini M, et al. First nationwide antimicrobial susceptibility surveillance for Neisseria gonorrhoeae in Brazil, 2015-16. J Antimicrob Chemother [Internet]. 2018 Jul [cited 2020 0ct 2]; 73(7):1854-61. Available from: https://doi.org/10.1093/jac/dky090

58. Ministério da Saúde (BR). Secretaria de Ciência, Tecnologia e Insumos Estratégicos. Comissão Nacional de Incorporação de Tecnologias no SUS (Conitec). Ceftriaxona 500mg para tratamento da Neisseria gonorrhoeae resistente à ciprofloxacina [Internet]. Brasília: Ministério da Saúde; 2015 [citado 2020 out 2]. (Relatório de Recomendação, n. 154). 29 p. Disponível em: http://conitec.gov.br/images/Relatorios/2015/Relatorio_Ceftriaxona_Gonorreia_final.pdf

59. Mitchell C, Prabhu M. Pelvic inflammatory disease: current concepts in pathogenesis, diagnosis and treatment. Infect Dis Clin North Am [Internet]. 2013 Dec [cited 2020 0ct 2]; 27(4):793-809. Available from: https://doi.org/10.1016/j.idc.2013.08.004

60. Public Health Agency of Canada - PHAC. Pelvic inflammatory disease (PID). In: PHAC. Updates to the Canadian guidelines on sexually transmitted infections [internet]. Ottawa: PHAC; 2008 [cited $2020 \mathrm{Jul} 22$ ]. 436 p. Available from: https://hs. mcmaster.ca/medicine/infectious_diseases/residents/docs/Canadian-STI-Guidelines2008.pdf

61. Jennings LK, Krywko DM. Pelvic inflammatory disease (PID). Stat Pearls [Internet]. 2020 Jun [cited 2020 Jul 22]. Available from: https:// www.ncbi.nlm.nih.gov/books/NBK499959/
62. Safrai M, Rottenstreich A, Shushan A, Gilad $\mathrm{R}$, Benshushan A, Levin G. Risk factors for recurrent Pelvic Inflammatory Disease. Eur J Obstet Gynecol Reprod Biol [Internet]. 2020 Jan [cited 2020 0ct 2]; 244:40-4. Available from: https://doi.org/10.1016/j.ejogrb.2019.11.004

63. Brasil. Ministério da Saúde. Portaria MS/SVS n ${ }^{0} 33$, de 14de julho de 2005. Inclui doenças à relação de notificação compulsória, define agravos de notificação imediata e a relação dos resultados laboratoriais que devem ser notificados pelos Laboratórios de Referência Nacional ou Regional [Internet]. Diário Oficial da União, Brasília (DF), 2005 jul 15 [citado 2020 out 2]; Seção 1:111. Disponível em: http://bvsms.saude.gov.br/ bvs/saudelegis/svs/2005/prt0033_14_07_2005.html

64. Wiesenfeld HC. Screening for Chlamydia trachomatis infections in women. NEngl J Med [Internet]. 2017 Feb [cited 2020 0ct 2]; 376(8):765-73. Available from: https://doi.org/10.1056/nejmcp1412935

65. Pinto-Sander N, Soni S. Mycoplasma genitalium infection. BMJ [Internet]. 2019 Oct [cited 2020 0ct 2]; 367:15820. Available from: https://doi.org/10.1136/bmj.15820

66. Greydanus DE, Dodich C. Pelvic inflammatory disease in the adolescent: a poignant, perplexing, potentially preventable problem for patients and physicians. Curr Opin Pediatr [Internet]. $2015 \mathrm{Feb}$ [cited 2020 0ct 2]; 27(1):92-9. Available from: https://doi.org/10.1097/mop.0000000000000183

67. Solomon M, Tuchman L, Hayes K, Badolato G, Goyal MK. Pelvic inflammatory disease in a pediatric emergency department: epidemiology and treatment. Pediatr Emerg Care [Internet]. 2019 Jun [cited 2020 0ct 2]; 35(6):389-90. Available from: https://doi.org/10.1097/pec.0000000000001148 


\section{Abstract}

Pelvic Inflammatory Disease is a topic included in the Clinical Protocol and Therapeutic Guidelines for Comprehensive Care for People with Sexually Transmitted Infections, published by the Brazilian Ministry of Health in 2020. Pelvic inflammatory disease is an acute infection of the upper female genital tract due to canalicular spread of endogenous cervicovaginal microorganisms, in particular sexually transmitted organisms. Standing out among the etiological agents involved are Chlamydia trachomatis and Neisseria gonorrhoeae. The most important sequels are chronic pelvic pain, infertility and ectopic pregnancy. Clinical diagnosis is the most important practical approach. Antibiotic treatment should start immediately upon clinical suspicion. The article contains guidance for health service managers and health professionals on diagnostic tests, treatment, follow-up, counseling, notification, handling of sexual partnerships and special populations. In view of increased availability of the molecular biology technique in Brazil, C. trachomatis and N. gonorrhoeae screening is recommended as a disease prevention strategy.

Keywords: Pelvic Infection; Pelvic Pain; Pregnancy, Ectopic; Infertility; Chlamydia trachomatis; Neisseria gonorrboeae.

\section{Resumen}

El tema de la enfermedad inflamatoria pélvica está incluido en el Protocolo Clínico y Directrices Terapéuticas para la Atención Integral para Personas con Infecciones de Transmisión Sexual, publicado por el Ministerio de Salud de Brasil en 2020. La enfermedad inflamatoria pélvica es una infección aguda del tracto genital superior femenino resultante del ascenso canalicular de microorganismos cervicovaginales endógenos y, principalmente, los de transmisión sexual. Entre los agentes etiológicos involucrados, se destacan Chlamydia trachomatis y Neisseria gonorrboeae. Las secuelas más importantes son: dolor pélvico crónico, infertilidad y embarazo ectópico. El diagnóstico clínico es el enfoque práctico más importante. El tratamiento con antibiótico debe iniciarse inmediatamente ante la sospecha clínica. Se describen pautas para gerentes y profesionales de la salud sobre pruebas de diagnóstico, tratamiento, seguimiento, asesoramiento, notificación, manejo de parejas sexuales y poblaciones especiales. Con la mayor disponibilidad de la técnica de biología molecular, se recomienda la detección de C. trachomatis y N. gonorrboeae como estrategia preventiva para la enfermedad.

Palabras clave: Infección Pélvica; Dolor Pélvico; Embarazo Ectópico; Infertilidad; Chlamydia trachomatis; Neisseria gonorrboeae.

Recebido em 21/07/2020

Aprovado em 16/09/2020 


\section{Errata}

No artigo "Protocolo Brasileiro para Infecções Sexualmente Transmissíveis 2020: doença inflamatória pélvica”, doi: 10.1590/\$1679-4974202100011.esp1, Figura 1 - Agentes etiológicos de doença inflamatória pélvica, publicado no periódico Epidemiologia e Serviços de Saúde, v. 30(Esp.1):1-14, na página 3:

Onde se lia:

\begin{tabular}{|c|c|}
\hline Manifestações clínicas de imunodeficiência moderada & Agentes etiológicos \\
\hline \multirow{4}{*}{ Microrganismos sexualmente transmissíveis } & Chlamydia trachomatis \\
\hline & Neisseria gonorrhoeae \\
\hline & Mycoplasma genitalium \\
\hline & Trichomonas vaginalis \\
\hline \multirow{10}{*}{ Microrganismos não sexualmente transmissíveis } & Mycoplasma hominis \\
\hline & Ureaplasma urealyticum \\
\hline & Bacteroides spp. e fragilis \\
\hline & Peptoestreptococcus spp. \\
\hline & Prevotella spp. \\
\hline & Escherichia coli \\
\hline & Gardnerella vaginalis \\
\hline & Haemophilus influenzae \\
\hline & Streptococcus spp. e agalactieae \\
\hline & Atopobium vaginae \\
\hline
\end{tabular}

Leia-se:

\begin{tabular}{|c|c|}
\hline Origem dos microrganismos & Agentes etiológicos \\
\hline \multirow{4}{*}{ Microrganismos sexualmente transmissíveis } & Chlamydia trachomatis \\
\hline & Neisseria gonorrhoeae \\
\hline & Mycoplasma genitalium \\
\hline & Trichomonas vaginalis \\
\hline \multirow{10}{*}{ Microrganismos não sexualmente transmissíveis } & Mycoplasma hominis \\
\hline & Ureaplasma urealyticum \\
\hline & Bacteroides spp. e fragilis \\
\hline & Peptoestreptococcus spp. \\
\hline & Prevotella spp. \\
\hline & Escherichia coli \\
\hline & Gardnerella vaginalis \\
\hline & Haemophilus influenzae \\
\hline & Streptococcus spp. e agalactieae \\
\hline & Atopobium vaginae \\
\hline
\end{tabular}

